\title{
The circular chromatic index of flower snarks
}

\author{
Mohammad Ghebleh \\ Department of Mathematics \\ Simon Fraser University \\ 8888 University Drive \\ Burnaby, BC, V5A 1S6, Canada \\ mghebleh@sfu.ca \\ Serguei Norine \\ School of Mathematics \\ Georgia Institute of Technology \\ Atlanta, GA 30332, USA \\ snorine@math.gatech.edu
}

\author{
Daniel Král'* \\ Department of Applied Mathematics and \\ Institute for Theoretical Computer Science ${ }^{\dagger}$ \\ Charles University \\ Malostranské náměstí 25 \\ 11800 Prague, Czech Republic \\ kral@kam.mff.cuni.cz \\ Robin Thomas ${ }^{\S}$ \\ School of Mathematics \\ Georgia Institute of Technology \\ Atlanta, GA 30332, USA \\ thomas@math.gatech.edu
}

Submitted: Oct 10, 2006; Accepted: Nov 6, 2006; Published: Nov 17, 2006

Mathematics Subject Classification: 05C15

\begin{abstract}
We determine the circular chromatic index of flower snarks, by showing that $\chi_{c}^{\prime}\left(F_{3}\right)=7 / 2, \chi_{c}^{\prime}\left(F_{5}\right)=17 / 5$ and $\chi_{c}^{\prime}\left(F_{k}\right)=10 / 3$ for every odd integer $k \geq 7$, where $F_{k}$ denotes the flower snark on $4 k$ vertices.
\end{abstract}

\section{Introduction}

All graphs in this paper are finite and simple. A graph is $k$-edge-colorable if its edges can be colored using $k$ colors in such a way that no two adjacent edges receive the same color. By a classical theorem of Vizing [11] every cubic graph is 4-edge-colorable, and hence cubic graphs fall into two categories: those that are 3-edge-colorable, and those that require four colors. Those of the latter kind that satisfy a mild connectivity requirement (cyclic 4-edge-connectivity) are called snarks. Snarks are of great interest $[2,4,5,12]$ because

\footnotetext{
${ }^{*}$ The author was a postdoctoral fellow at TU Berlin from October 2004 to July 2005 within the framework of the European training network COMBSTRU.

${ }^{\dagger}$ Institute for Theoretical Computer Science (ITI) is supported by Ministry of Education of Czech Republic as project $1 \mathrm{M} 0545$.

${ }_{\ddagger}^{\ddagger}$ Partially supported by NSF Grant No. 0200595.

$\S$ Partially supported by NSF Grants No. 0200595 and 0354742.
} 
the non-existence of planar snarks is equivalent to the Four Color Theorem [9], and it is known that a minimal counterexample to several important conjectures must be a snark, see e.g. $[5,13]$.

Are some snarks closer to being 3-edge-colorable than others? This question can be made precise using the concept of circular coloring, introduced by Vince [10] under the name of star coloring. For $r>0$, an $r$-circular edge-coloring of a graph $G$ is a mapping $c: E(G) \rightarrow[0, r)$ such that $1 \leq|c(e)-c(f)| \leq r-1$ for every two adjacent edges $e$ and $f$ of $G$. The circular chromatic index of $G$ is the infimum (in fact, the minimum) of all $r>0$ such that $G$ has an $r$-circular edge-coloring. The circular chromatic index of $G$ is denoted by $\chi_{c}^{\prime}(G)$. It is not hard to show that the chromatic index of $G$ (the least $k$ such that $G$ is $k$-edge-colorable) is equal to $\left\lceil\chi_{c}^{\prime}(G)\right\rceil$, and hence the circular chromatic index provides a finer measure of edge-colorability than the chromatic index. We refer the reader to the survey $[14,15]$ for more details on circular colorings of graphs.

Zhu [14] asked whether there exists a snark with circular chromatic index four. Afshani et al. [1] answered the question in the negative by showing that the circular chromatic index of every bridgeless cubic graph is at most 11/3. The bound is tight as witnessed by the Petersen graph. However, the Petersen graph is the only known bridgeless cubic graph with circular chromatic index equal to $11 / 3$, and so it seems natural to look for other examples among known families of snarks.

There is another result on circular edge colorings that motivated our work. Kochol [8] disproved the conjecture of Jaeger and Swart [3] that the girth of every snark is bounded by an absolute constant by constructing snarks of arbitrarily high girth. However, the conjecture holds in an approximate sense when relaxed to circular colorings: Kaiser et al. [6] proved that for every $\varepsilon>0$ there exists an integer $g$ such that every cubic bridgeless graph of girth at least $g$ has circular chromatic index at most $3+\varepsilon$. This result was extended in [7] to graphs with arbitrary maximum degree.

It is natural to ask whether perhaps the same conclusion (at least for cubic graphs) holds under the weaker assumption that the graph have odd girth at least $g$, i.e., that the graph have no odd cycle of length strictly less than $g$. We show that this is not the case by proving that the family of snarks known as flower snarks provide a counterexample. We were actually able to compute the circular chromatic index of flower snarks exactly. Let us recall that for an odd integer $k \geq 3$ the flower snark $F_{k}$, is the following graph [4]: the vertex set of $F_{k}$ consists of $4 k$ vertices $v_{1}, \ldots, v_{k}$ and $u_{1}^{1}, u_{1}^{2}, u_{1}^{3}, \ldots, u_{k}^{1}, u_{k}^{2}, u_{k}^{3}$. The graph is comprised of a cycle $u_{1}^{1} \cdots u_{k}^{1}$ of length $k$ and a cycle $u_{1}^{2} \cdots u_{k}^{2} u_{1}^{3} \cdots u_{k}^{3}$ of length $2 k$, and in addition, each vertex $v_{i}$ is adjacent to $u_{i}^{1}, u_{i}^{2}$ and $u_{i}^{3}$.

\section{General bound}

Let $\varepsilon>0$ and set $r=10 / 3-\varepsilon$. We show that no flower snark has an $r$-circular edgecoloring. The elements of $[0, r)$ are referred to as colors. For $0 \leq a \leq b \leq r$, define $\rho(a, b)=\rho(b, a)$ to be $\min \{b-a, r+a-b\}$, i.e., $\rho(x, y)$ is the distance between $x$ and $y$ on a circle of perimeter $r$. If $b-a<r+a-b$, then we say that $b$ follows $a$; if $b-a>r+a-b$, then $a$ follows $b$. Two colors $x$ and $y$ are close if $\rho(x, y)<2 / 3$ and they are far apart if 
$\rho(x, y)>2 / 3$. A sequence $\left(c_{0}, c_{1}, c_{2}\right)$ of colors is of type $A$ if $c_{0}, c_{1}$ and $c_{2}$ are pairwise far apart, and it is of type $B$ if two of the colors are close and the remaining one is far apart from both of the other two. A sequence $\left(c_{0}, c_{1}, c_{2}\right)$ of type $\mathrm{A}$ has positive sign if $0 \leq c_{i} \leq c_{i+1} \leq c_{i+2}<r$ for some $i=0,1,2$, where index arithmetic is taken modulo 3 , and it has negative sign otherwise. In other words, the sequence $\left(c_{0}, c_{1}, c_{2}\right)$ has positive sign if it can be obtained from the sorted sequence comprised of $c_{0}, c_{1}$ and $c_{2}$ by an even number of transpositions. We now define signs for sequences of type B. Let $\left(c_{0}, c_{1}, c_{2}\right)$ be a sequence of colors of type $\mathrm{B}$ and let $i, j, k$ be such that $\{i, j, k\}=\{0,1,2\}$ and $c_{i}$ and $c_{j}$ are close. The sequence $\left(c_{0}, c_{1}, c_{2}\right)$ has positive sign if the color $c_{k}$ follows both $c_{i}$ and $c_{j}$, and we say that it has negative sign if $c_{i}$ and $c_{j}$ both follow the color $c_{k}$. Note that for sequences of type B the sign need not be defined and in case it is defined, it does not depend on the order of the elements in the sequence.

Similarly as in the original proof that flower snarks are not 3-edge-colored, a certain parity argument is also involved in our proof. The following lemma captures this:

Lemma 1. Let $c$ be an $r$-circular edge-coloring of a cubic graph $G$ for $r=10 / 3-\varepsilon$ with $\varepsilon>0$. Let $v$ be a vertex of $G, u_{1}, u_{2}$ and $u_{3}$ be its neighbors, and $e_{i}$ and $f_{i}$ edges incident with $u_{i}$ but not with $v$ (for $i \in\{1,2,3\}$ ). If all the edges $e_{1}, e_{2}, e_{3}, f_{1}, f_{2}$ and $f_{3}$ are distinct, then the following holds: either $\left(c\left(e_{1}\right), c\left(e_{2}\right), c\left(e_{3}\right)\right)$ and $\left(c\left(f_{1}\right), c\left(f_{2}\right), c\left(f_{3}\right)\right)$ are both of type $A$ and have the same sign or the two sequences are both of type $B$ and have different signs (in particular, the signs of both of them are defined).

Proof. For every color $a$ let $I(a)=\{a+x: 1 \leq x \leq 4 / 3-\epsilon\}$ and $J(a)=\{a-x: 1 \leq x \leq$ $4 / 3-\epsilon\}$, where addition and subtraction is modulo $r$. Note that if the edges $e, e^{\prime}$ and $e^{\prime \prime}$ are distinct and share a vertex, then exactly one of $c\left(e^{\prime}\right)$ and $c\left(e^{\prime \prime}\right)$ belongs to $I(c(e))$ and the other belongs to $J(c(e))$.

Let $c_{i}=c\left(v u_{i}\right), a_{i}=c\left(e_{i}\right)$ and $b_{i}=c\left(f_{i}\right)$ for $i=1,2,3$. Since the edges $v u_{1}, v u_{2}$ and $v u_{3}$ share a vertex, the sequence $\left(c_{1}, c_{2}, c_{3}\right)$ is of type A. By the symmetry, we may assume that it has positive sign, i.e., $c_{1} \in I\left(c_{3}\right) \cap J\left(c_{2}\right), c_{2} \in I\left(c_{1}\right) \cap J\left(c_{3}\right)$, and $c_{3} \in I\left(c_{2}\right) \cap J\left(c_{1}\right)$. Hence, any two colors in $I\left(c_{3}\right) \cup J\left(c_{2}\right)$ are close, and the same holds for $I\left(c_{1}\right) \cup J\left(c_{3}\right)$ and $I\left(c_{2}\right) \cup J\left(c_{1}\right)$. By reversing the roles of $I$ and $J$, we may assume that $a_{1} \in I\left(c_{1}\right)$ and $b_{1} \in J\left(c_{1}\right)$. Assume first that $a_{1}, a_{2}$ and $a_{3}$ are pairwise far apart. Then, $a_{3} \in I\left(c_{3}\right)$ (because $a_{3} \notin J\left(c_{3}\right)$ since it is far apart from $a_{1}$ ), and similarly $a_{2} \in I\left(c_{2}\right)$. Consequently, $b_{3} \in J\left(c_{3}\right)$ and $b_{2} \in J\left(c_{2}\right)$. The circular intervals $I\left(c_{1}\right), I\left(c_{2}\right)$ and $I\left(c_{3}\right)$ are pairwise at distance at least $2 / 3$, and the same holds for $J\left(c_{1}\right), J\left(c_{2}\right)$ and $J\left(c_{3}\right)$. It follows that $\left(a_{1}, a_{2}, a_{3}\right)$ has positive sign, and that $\left(b_{1}, b_{2}, b_{3}\right)$ is of type $\mathrm{A}$ and it also has positive sign.

The other case to consider is that two of $a_{1}, a_{2}$ and $a_{3}$ are close. By the symmetry, we may assume that $a_{2}$ and $a_{3}$ are close, i.e., $a_{2} \in J\left(c_{2}\right)$ and $a_{3} \in I\left(c_{3}\right)$. Hence, $b_{2} \in I\left(c_{2}\right)$ and $b_{3} \in J\left(c_{3}\right)$. We have that every member of $I\left(c_{1}\right)$ follows every member of $I\left(c_{3}\right) \cup J\left(c_{2}\right)$, because $c_{1}$ is at distance one from one end of the circular interval $I\left(c_{1}\right)$, and each of the circular intervals involved has length $1 / 3-\epsilon$. Thus $a_{1}$ follows $a_{2}$ and $a_{3}$. Since $b_{1}, b_{2} \in I\left(c_{2}\right) \cup J\left(c_{1}\right)$, we deduce that $b_{1}$ and $b_{2}$ are close, and it follows similarly as above that both $b_{1}$ and $b_{2}$ follow $b_{3}$, as desired. We conclude that both $\left(a_{1}, a_{2}, a_{3}\right)$ and $\left(b_{1}, b_{2}, b_{3}\right)$ are of type $\mathrm{B}$ and have different signs. 


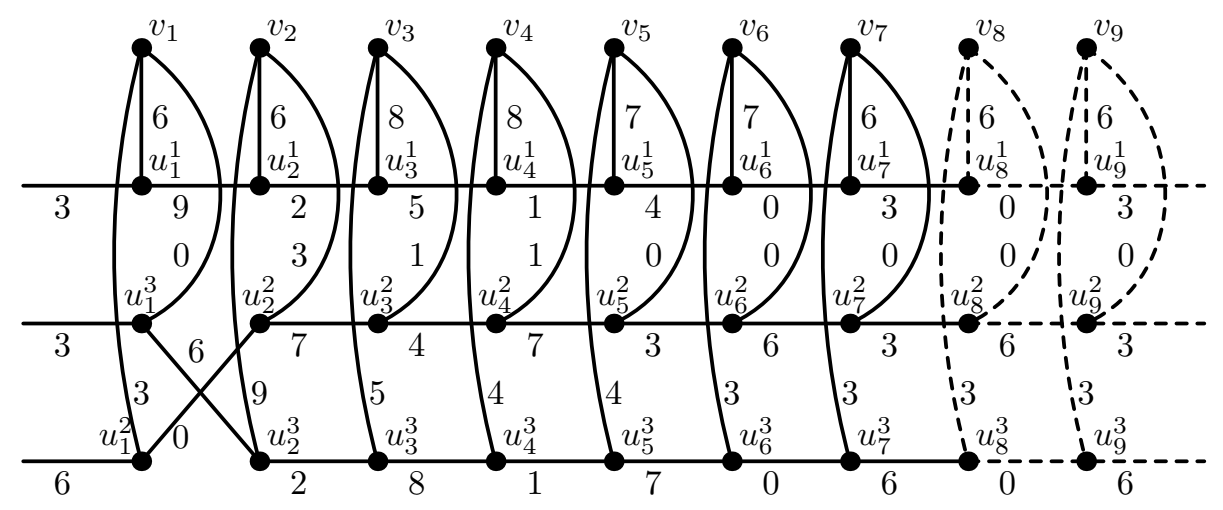

Figure 1: The construction of a 10/3-circular edge-coloring of large flower snarks. The colors of the edges are multiplied by three in the figure.

We are now ready to deduce the lower bound on the circular chromatic indices of flower snarks:

Theorem 1. For every $t \geq 1$, the circular chromatic index of the flower snark $F_{2 t+1}$ is at least $10 / 3$. Moreover, if $t \geq 3$, then $\chi_{c}^{\prime}\left(F_{2 t+1}\right)=10 / 3$.

Proof. Let $t \geq 1$ and suppose for a contradiction that $c$ is an $r$-circular edge-coloring of $F_{2 t+1}$ with $r<10 / 3$. We repeatedly apply Lemma 1 with $v=v_{i}, e_{1}=u_{i}^{1} u_{i-1}^{1}, e_{2}=u_{i}^{2} u_{i-1}^{2}$, $e_{3}=u_{i}^{3} u_{i-1}^{3}, f_{1}=u_{i}^{1} u_{i+1}^{1}, f_{2}=u_{i}^{2} u_{i+1}^{2}$ and $f_{3}=u_{i}^{3} u_{i+1}^{3}$ for $i=1, \ldots, 2 t+1$ (we set $u_{0}^{1}=$ $u_{2 t+1}^{1}, u_{2 t+2}^{1}=u_{1}^{1}, u_{0}^{2}=u_{2 t+1}^{3}, u_{2 t+2}^{2}=u_{1}^{3}, u_{0}^{3}=u_{2 t+1}^{2}$ and $u_{2 t+2}^{3}=u_{1}^{2}$ where appropriate). In this way, we conclude that either the sequences $\left(c\left(u_{2 t+1}^{1} u_{1}^{1}\right), c\left(u_{2 t+1}^{3} u_{1}^{2}\right), c\left(u_{2 t+1}^{2} u_{1}^{3}\right)\right)$ and $\left(c\left(u_{2 t+1}^{1} u_{1}^{1}\right), c\left(u_{2 t+1}^{2} u_{1}^{3}\right), c\left(u_{2 t+1}^{3} u_{1}^{2}\right)\right)$ are both of type $\mathrm{A}$ and have the same sign (which is impossible since they differ by a single transposition) or they are both of type B and have different signs (which is again impossible by the definition of the sign in this case). This establishes that $\chi_{c}^{\prime}\left(F_{2 t+1}\right) \geq 10 / 3$.

Figure 1 shows the construction of a 10/3-circular edge-coloring of $F_{2 t+1}$ for $t \geq 3$. The edges of the snark $F_{2 t+1}$ incident with the vertices $v_{1}, \ldots, v_{7}, u_{1}^{1}, \ldots, u_{7}^{1}, u_{1}^{2}, \ldots, u_{7}^{2}$ and $u_{1}^{3}, \ldots, u_{7}^{3}$ are always colored as in the figure. The remaining edges of $F_{2 t+1}$ are colored using the pattern shown on the dashed edges. Note that the colors in the figure are multiplied by three for better clarity.

\section{The flower snark $F_{3}$}

An easy consequence of Lemma 1.3 of [14] is the following:

Proposition 1. Let $G$ be a graph. If $\chi_{c}^{\prime}(G)=p / q$ (where $p$ and $q$ are relatively prime), then there exists a $p / q$-circular edge-coloring of $G$ with colors $0 / q, \ldots,(p-1) / q$ only and each of these colors is assigned to at least one edge of $G$.

Since the edges colored with the colors $0 / q, \ldots,(q-1) / q$ must form a matching in $G$, we obtain the following from Proposition 1: 


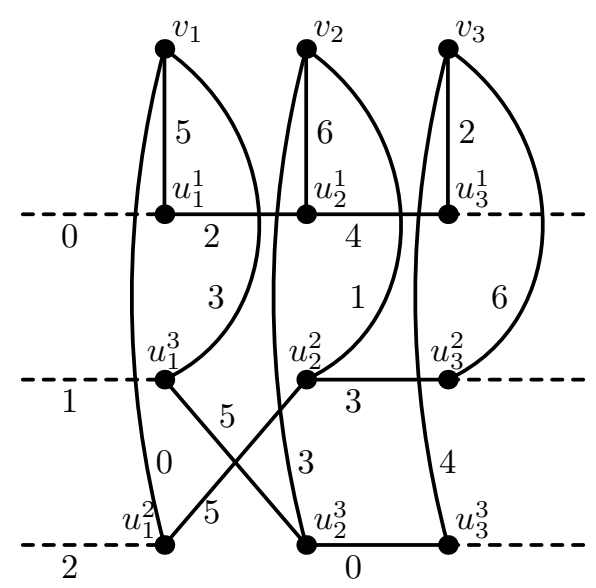

Figure 2: A 7/2-circular edge-coloring of $F_{3}$. The dashed edges wrap "around" the figure. The colors of all the edges are multiplied by two.

Proposition 2. Let $G$ be a graph. If $\chi_{c}^{\prime}(G)=p / q$ (where $p$ and $q$ are relatively prime), then $q$ is at most the cardinality of a maximum matching of $G$.

We are now ready to determine the circular chromatic index of $F_{3}$ :

Theorem 2. The circular chromatic index of $F_{3}$ is $7 / 2$.

Proof. A 7/2-circular edge-coloring of $F_{3}$ can be found in Figure 2. By Theorem 1, the circular chromatic index of $F_{3}$ is at least 10/3. By Proposition 2, $\chi_{c}^{\prime}\left(F_{3}\right) \in\{10 / 3,17 / 5,7 / 2\}$.

Assume that $\chi_{c}^{\prime}\left(F_{3}\right)=10 / 3$. By Proposition 1, there exists a 10/3-circular edgecoloring $c$ of $F_{3}$ which is onto the set $\{0 / 3, \ldots, 9 / 3\}$. By the pigeon-hole principle, at least one of the colors is assigned to a single edge of $F_{3}$ (the size of $F_{3}$ is 18). Assume that $\left|c^{-1}(9 / 3)\right|=1$. Observe that all the three sets $c^{-1}(\{0 / 3,1 / 3,2 / 3\}), c^{-1}(\{3 / 3,4 / 3,5 / 3\})$ and $c^{-1}(\{6 / 3,7 / 3,8 / 3\})$ of edges form matchings in $F_{3}$ and at least two of them are matchings of size six, i.e., perfect matchings. However, $F_{3}$ does not contain two disjoint perfect matchings since it is not 3-edge-colorable.

Assume that $\chi_{c}^{\prime}\left(F_{3}\right)=17 / 5$. By Proposition 1, there exists a $17 / 5$-circular edgecoloring $c$ of $F_{3}$ which maps onto the set $\{0 / 5, \ldots, 16 / 5\}$. Observe that each of the colors is assigned to a single edge of $F_{3}$ except for one of the colors which is assigned to two edges of $F_{3}$. Assume that the exceptional color is $1 / 5$. Both the sets $c^{-1}(\{0 / 5,1 / 5,2 / 5,3 / 5,4 / 5\})$ and $c^{-1}(\{1 / 5,2 / 5,3 / 5,4 / 5,5 / 5\})$ of edges of $F_{3}$ are perfect matchings, say $M_{1}$ and $M_{2}$. By their choice, $\left|M_{1} \cap M_{2}\right|=5$. However, two perfect matchings of a simple graph cannot differ at a single edge.

\section{The flower snark $F_{5}$}

A construction of 17/5-circular edge coloring of $F_{5}$ depicted in Figure 3 shows that $\chi_{c}^{\prime}\left(F_{5}\right) \leq 17 / 5$. We were not able to provide the matching lower bound without the 


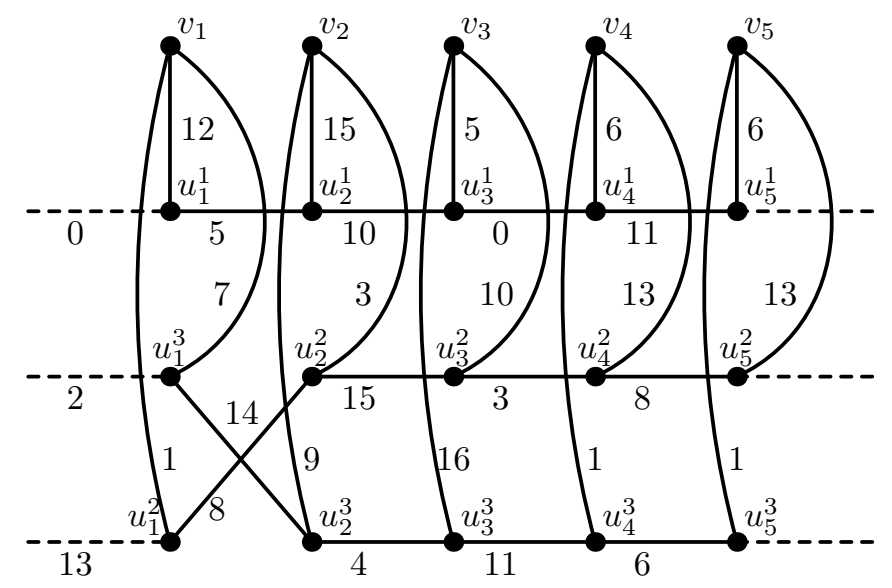

Figure 3: An 17/5-circular edge-coloring of $F_{5}$. The dashed edges wrap "around" the figure. The colors of all the edges are multiplied by five.

assistance of a computer. By Theorem 1 and Proposition 2, it is enough to exclude the cases that $\chi_{c}^{\prime}\left(F_{5}\right)=10 / 3$ or $\chi_{c}^{\prime}\left(F_{5}\right)=27 / 8$. A brute force algorithm for finding such an edge-coloring will be too slow. Therefore, we designed a faster algorithm for verifying the existence of $p / q$-circular edge-coloring of $F_{2 t+1}$ based on the following idea: first, we construct an auxiliary graph of order $p^{3}$. The vertices of this graph are all the sequences of colors of length three. Two such sequences $\left(a_{1}, a_{2}, a_{3}\right)$ and $\left(a_{1}^{\prime}, a_{2}^{\prime}, a_{3}^{\prime}\right)$ are joined by an edge if it is possible to extend the partial coloring $c\left(u_{1}^{i} u_{2}^{i}\right)=a_{i}$ and $c\left(u_{2}^{i} u_{3}^{i}\right)=a_{i}^{\prime}$ to the three edges incident with the vertex $v_{2}$. It is not hard to observe that $F_{2 t+1}$ has a $p / q$ circular coloring if and only if the auxiliary graph contains a walk of length $2 t+1$ from a vertex $\left(a_{1}, a_{2}, a_{3}\right)$ to a vertex $\left(a_{1}, a_{3}, a_{2}\right)$ for some choice of $a_{1}, a_{2}, a_{3} \in\{0 / q, \ldots,(p-1) / q\}$. Once the auxiliary graph is constructed (which may be done quite fast even if the brute force algorithm for determining the adjacency of its vertices is used), the existence of the walk can be decided in time linear in the size of the auxiliary graph. In this way, we verified that $F_{5}$ has neither 10/3-circular nor 27/8-circular edge-coloring. Based on the discussion of the previous two sections, we conclude:

Theorem 3. The following holds for every $t \geq 1$ :

$$
\chi_{c}^{\prime}\left(F_{2 t+1}\right)=\left\{\begin{array}{cl}
7 / 2 & \text { if } t=1, \\
17 / 5 & \text { if } t=2, \text { and } \\
10 / 3 & \text { otherwise }
\end{array}\right.
$$

\section{References}

[1] P. Afshani, M. Ghandehari, M. Ghandehari, H. Hatami, R. Tusserkani, X. ZHU: Circular chromatic index of graphs of maximum degree 3, J. Graph Theory 49(4) (2005), 325-335. 
[2] D. BlanušA: Problem cetiruju boja, Glasnik Mat. Fiz. Astr. Ser. II. 1 (1946), 31-42.

[3] F. Jaeger, T. Swart: Conjecture 1, in: Combinatorics 79 (M. Deza, I. G. Rosenberg, eds.), Ann. Discrete Math. Vol. 9, North-Holland, Amsterdam, 1980, p. 305.

[4] R. IsAaCs: Infinite families of nontrivial trivalent graphs which are not Tait colorable, Amer. Math. Monthly 82 (1975), 221-239.

[5] T. R. Jensen, B. Toft: Graph coloring problems, Wiley Interscience, 1995, New York.

[6] T. Kaiser, D. KráL', R. Škrekovski: A revival of the girth conjecture, J. Combin. Theory Ser. B 92 (2004), 41-53.

[7] T. Kaiser, D. Král', R. Škrekovski, X. Zhu: The circular chromatic index of graphs of high girth, to appear in J. Combin. Theory Ser. B.

[8] M. Kochol: Snarks without small cycles, J. Combin. Theory Ser. B 67 (1996), $34-47$.

[9] P. G. TAIT: Remarks on the colouring of maps, Proc. Roy. Soc. Edingburg 10 (1880), 501-503.

[10] A. VincE: Star chromatic number, J. Graph Theory 12 (1988), 551-559.

[11] V. G. VIZING: On an estimate of the chromatic class of a p-graph (in Russian), Diskret. Analiz. 3 (1964), 24-30.

[12] G. Szekeres: Polyhedral decompositions of cubic graphs, Bull. Austral. Math. Soc. 8 (1973), 367-387.

[13] C.-Q. Zhang: Integer flows and cycle covers of graphs, Monographs and Textbooks in Pure and Applied Mathematics 205, Marcel Dekker, 1997, New York.

[14] X. ZHU: Circular chromatic number: a survey, Discrete Math. 229 (2001), 371-410.

[15] X. ZHU: Recent developments in circular colorings of graphs, in M. Klazar, J. Kratochvíl, J. Matoušek, R. Thomas, P. Valtr (eds.): Topics in Discrete Mathematics, Springer, 2006, 497-550. 